\title{
Recovery of the boundary data for a linear second order elliptic problem with a nonlocal boundary condition
}

\author{
Hennie De Schepper* Marián Slodička*
}

(Received 6 June 2000)

\begin{abstract}
We study a linear elliptic partial differential equation of second order in a bounded domain $\Omega \subset \mathrm{R}^{N}$, with nonstandard boundary conditions on a part $\Gamma$ of the boundary $\partial \Omega$. Here, neither the solution nor its normal derivative are prescribed pointwise. Instead, the average of the solution over $\Gamma$ is given and the normal derivative along
\end{abstract}

*Department of Mathematical Analysis, Ghent University, Galglaan 2, B-9000 Ghent, BELGIUM. mailto:hds@cage.rug.ac.be and mailto:ms@cage.rug.ac.be

${ }^{0}$ See http: //anziamj . austms .org.au/V42/CTAC99/Desc for this article and ancillary services, (C) Austral. Mathematical Soc. 2000. Published 27 Nov 2000. 
$\Gamma$ has to follow a prescribed shape function, apart from an additive (unknown) constant. We prove the well-posedness of the problem and provide a method for the recovery of the unknown boundary data.

\section{Contents}

1 Introduction

C519

2 Problem setting

C521

3 Uniqueness and existence

C523

4 Numerical experiments

C528

4.1 First example

C528

4.2 Second example

C531

References

C535

\section{Introduction}

The problem of parameter identification from nonstandard boundary conditions (BCs) in boundary value problems (BVPs), originating from various engineering disciplines, is of growing interest. Standard (Dirichlet, Neumann 
and Robin type) BCs which are prescribed pointwise are not always adequate, as it depends on the physical context which data can be measured at the boundary of the physical domain. In some cases, it is not possible to prescribe the solution $u$ (pressure, temperature, ... ) or the flux $\boldsymbol{q} \cdot \boldsymbol{\nu}$ pointwise, because only the average value of the solution or of the total flux can be measured along some boundary part $\Gamma$. In case that $\int_{\Gamma} u$ or $\int_{\Gamma} \boldsymbol{q} \cdot \boldsymbol{\nu}$ is prescribed, we are dealing with a nonlocal $\mathrm{BC}$. This type of $\mathrm{BC}$ is not sufficient in order to determine the solution uniquely. One needs some additional information, resulting from other physical arguments, e.g., about the shape of $u$ or of $\boldsymbol{q} \cdot \boldsymbol{\nu}$ along $\Gamma$. For instance, nonstandard BCs of the type

$$
u=c \in \mathrm{R} \text { (unknown) on } \Gamma, \quad \int_{\Gamma}-K \nabla u \cdot \boldsymbol{\nu}=s \text { (given), }
$$

appear in the modelling of soil venting (cf. [2]). Here $u$ stands for the squared air pressure, $K$ denotes the air-transmissivity of the soil matrix, and $\Gamma$ represents the boundary of an "extraction well" or pump. The BCs can be interpreted as follows. On one hand, the total amount of extracted air can be measured; on the other hand, one can assume the air pressure along the boundary of an extraction well to be constant, however unknown. The existence of a solution for BVPs with BCs including the type (1) has been proved in $[2]$.

The dual type of BCs, viz

$$
\boldsymbol{q} \cdot \boldsymbol{\nu}=c \in \mathrm{R} \text { (unknown) on } \Gamma, \quad \int_{\Gamma} u=U \text { (given), }
$$


appears in the modelling of some electromagnetic field problems in electric machinery (cf. [4]). Moreover, it also has a physical interpretation in the above mentioned context of soil venting. For a single well experiment in a homogeneous soil matrix one may assume that the inflow of air into the well tube is constant, but unknown, while the average pressure at the well is given. Here, some pressure variations along the boundary of the well are allowed for.

The main goal of this paper is to study a class of 2nd order linear elliptic PDEs with BCs of a type including (2). We prove the existence and the uniqueness of the solution, and we outline a method for constructing it in terms of the solution of some auxiliary BVPs with standard (i.e. pointwise) BCs. In particular, the unknown flux and the trace of the solution at $\Gamma$ are determined.

\section{Problem setting}

Let $\Omega \subset \mathrm{R}^{N}$ be an open bounded domain with a Lipschitz continuous boundary $\partial \Omega$, which consists of a finite number of disjoint parts, i.e., $\partial \Omega=$ $\Gamma_{D} \cup \Gamma_{N} \cup_{i=1}^{n} \Gamma_{i}$. 
We consider the following BVP:

$$
\begin{aligned}
\nabla \cdot\left(-\boldsymbol{A}_{\text {dif }} \nabla u-\boldsymbol{a}_{\text {con }} u\right)+a_{\text {sou }} u & =f & & \text { in } \Omega \\
u & =g_{\text {Dir }} & & \text { on } \Gamma_{D} \\
\left(-\boldsymbol{A}_{\text {dif }} \nabla u-\boldsymbol{a}_{\text {con }} u\right) \cdot \boldsymbol{\nu}-g_{\text {Rob }} u & =g_{N e u} & & \text { on } \Gamma_{N} \\
\left(-\boldsymbol{A}_{\text {dif }} \nabla u-\boldsymbol{a}_{\text {con }} u\right) \cdot \boldsymbol{\nu} & =q_{i}+c_{i} & & \text { on } \Gamma_{i} \\
\int_{\Gamma_{i}} u & =U_{i} & &
\end{aligned}
$$

for a given set of values $U_{i} \in \mathrm{R}$ and a given set of shape functions $q_{i},(i=$ $1, \ldots, n)$. Here, the normal component of the flux $\boldsymbol{q}$ is prescribed on $\Gamma_{i}$ by the shape function $q_{i}$, up to an additive unknown real constant $c_{i}$, which has to be determined as a part of the problem.

Consider the space

$$
V=\left\{\varphi \in W^{1,2}(\Omega) \mid \varphi=0 \text { on } \Gamma_{D}\right\}
$$

where $W^{1,2}(\Omega)$ stands for the standard first-order Sobolev space on $\Omega$, endowed with the $\|\cdot\|_{1,2, \Omega}$-norm. We define the bilinear form $a$ on $V \times V$ by

$$
a(u, v)=\int_{\Omega}\left[\boldsymbol{A}_{d i f} \nabla u+\boldsymbol{a}_{c o n} u\right] \cdot \nabla v+\int_{\Omega} a_{\text {sou }} u v+\int_{\Gamma_{N}} g_{R o b} u v .
$$

We assume the form $a$ to be $V$-elliptic, i.e.,

$$
\exists C_{0}>0: a(w, w) \geq C_{0}\|w\|_{1,2, \Omega}^{2}, \quad \forall w \in V .
$$


For a set of conditions on the data functions $\boldsymbol{A}_{\text {dif }}, \boldsymbol{a}_{\text {con }}, a_{\text {sou }}$ and $g_{\text {Rob }}$, entering (3), which ensure the $V$-ellipticity of $a$, we may refer to $[1$, p.1227 and p.1245]. In addition, the functions $f, g_{D i r}, g_{N e u}$ and $q_{i}$ obey the conditions

$$
f \in L_{2}(\Omega), g_{D i r} \in L_{2}\left(\Gamma_{D}\right), g_{N e u} \in L_{2}\left(\Gamma_{N}\right) \text { and } q_{i} \in L_{2}\left(\Gamma_{i}\right), i=1, \ldots, n .
$$

\section{Uniqueness and existence}

First, we prove the uniqueness of the solution of the BVP (3). In turn, this uniqueness will be used in the proof of the existence theorem.

Theorem 1 (uniqueness) There exists at most one solution of the BVP (3).

Proof: Assume that there exist two solutions $u$ and $w$ of (3). Then, we subtract the corresponding PDEs, we multiply the result by $u-w$ and we integrate the equality obtained over $\Omega$. Applying Green's theorem, we have

$$
\begin{gathered}
\int_{\Omega}\left[\boldsymbol{A}_{d i f} \nabla(u-w)+\boldsymbol{a}_{c o n}(u-w)\right] \cdot \nabla(u-w)+\int_{\Omega} a_{\text {sou }}(u-w)^{2} \\
-\int_{\partial \Omega}\left[\boldsymbol{A}_{d i f} \nabla(u-w)+\boldsymbol{a}_{c o n}(u-w)\right] \cdot \boldsymbol{\nu}(u-w)=0 .
\end{gathered}
$$

The integral over $\partial \Omega$ can be simplified as follows. As $u-w=0$ on $\Gamma_{D}$ and as moreover from (3d) the normal component of the flux of $u-w$ on $\Gamma_{i}$ is 
constant, we have

$$
\begin{gathered}
\int_{\partial \Omega}\left[\boldsymbol{A}_{d i f} \nabla(u-w)+\boldsymbol{a}_{c o n}(u-w)\right] \cdot \boldsymbol{\nu}(u-w) \\
=-\int_{\Gamma_{N}} g_{R o b}(u-w)^{2}+\sum_{i=1}^{n}\left[\boldsymbol{A}_{d i f} \nabla(u-w)+\boldsymbol{a}_{c o n}(u-w)\right] \cdot \boldsymbol{\nu} \int_{\Gamma_{i}}(u-w) \\
=-\int_{\Gamma_{N}} g_{R o b}(u-w)^{2}
\end{gathered}
$$

where in the last equality we have invoked the nonlocal BC (3e). Consequently, (6) reduces to

$$
a(u-w, u-w)=0,
$$

from which we conclude that $u=w$ on account of the $V$-ellipticity (4).

We set up a constructive proof of the existence of a solution of the BVP (3). The ideas underlying the construction of the solution are as follows.

At each boundary part $\Gamma_{i},(i=1, \ldots, n)$, two conditions are imposed, one of them having a nonlocal character, the other containing an unknown parameter. When we omit the nonlocal (integral) side condition at each $\Gamma_{i}$ and we suppose the constants $c_{i}$ to be given, the resulting BVP is wellposed. We investigate the dependence of the corresponding solution on the constants $c_{i},(i=1, \ldots, n)$. This idea will be combined with the principle of linear superposition to find a set $\left(c_{1}, \ldots, c_{n}\right)$ for which also the nonlocal BCs at $\Gamma_{i}$ hold, $(i=1, \ldots, n)$. Thus, we consider two types of auxiliary problems. 
First, we take into account the source function $f$, the BCs on the Dirichlet and the Neumann part of $\partial \Omega$ and the shape functions $\Gamma_{i}, i=1, \ldots, n$ :

$$
\left\{\begin{aligned}
\nabla \cdot\left(-\boldsymbol{A}_{d i f} \nabla v-\boldsymbol{a}_{c o n} v\right)+a_{\text {sou }} v & =f & & \text { in } \Omega \\
v & =g_{D i r} & & \text { on } \Gamma_{D} \\
\left(-\boldsymbol{A}_{d i f} \nabla v-\boldsymbol{a}_{c o n} v\right) \cdot \boldsymbol{\nu}-g_{R o b} v & =g_{N e u} & & \text { on } \Gamma_{N} \\
\left(-\boldsymbol{A}_{d i f} \nabla v-\boldsymbol{a}_{c o n} v\right) \cdot \boldsymbol{\nu} & =q_{i} & & \text { on } \Gamma_{i}, i=1, \ldots, n .
\end{aligned}\right.
$$

Next, for $i=1, \ldots, n$, we introduce a function $z_{i}$ by:

$$
\left\{\begin{aligned}
\nabla \cdot\left(-\boldsymbol{A}_{d i f} \nabla z_{i}-\boldsymbol{a}_{\text {con }} z_{i}\right)+a_{\text {sou }} z_{i} & =0 & & \text { in } \Omega \\
z_{i} & =0 & & \text { on } \Gamma_{D} \\
\left(-\boldsymbol{A}_{d i f} \nabla z_{i}-\boldsymbol{a}_{\text {con }} z_{i}\right) \cdot \boldsymbol{\nu}-g_{\text {Rob }} z_{i} & =0 & & \text { on } \Gamma_{N} \\
\left(-\boldsymbol{A}_{d i f} \nabla z_{i}-\boldsymbol{a}_{\text {con }} z_{i}\right) \cdot \boldsymbol{\nu} & =1 & & \text { on } \Gamma_{i} \\
\left(-\boldsymbol{A}_{d i f} \nabla z_{i}-\boldsymbol{a}_{\text {con }} z_{i}\right) \cdot \boldsymbol{\nu} & =0 & & \text { on } \Gamma_{j}, j \neq i .
\end{aligned}\right.
$$

Each of these auxiliary problems has a unique solution (see, e.g., [3]).

Now, for any $\boldsymbol{\alpha}=\left(\alpha_{1}, \ldots, \alpha_{n}\right) \in \mathrm{R}^{n}$, we define the function $u_{\boldsymbol{\alpha}}$ as

$$
u_{\boldsymbol{\alpha}}=v+\sum_{i=1}^{n} \alpha_{i} z_{i}
$$

By the linearity of the BVPs (7)-(8), one easily gets that $u_{\boldsymbol{\alpha}}$, (9), solves the following problem:

$$
\left\{\begin{aligned}
\nabla \cdot\left(-\boldsymbol{A}_{d i f} \nabla u_{\boldsymbol{\alpha}}-\boldsymbol{a}_{\text {con }} u_{\boldsymbol{\alpha}}\right)+a_{\text {sou }} u_{\boldsymbol{\alpha}} & =f & & \text { in } \Omega \\
u_{\boldsymbol{\alpha}} & =g_{D i r} & & \text { on } \Gamma_{D} \\
\left(-\boldsymbol{A}_{d i f} \nabla u_{\boldsymbol{\alpha}}-\boldsymbol{a}_{\text {con }} u_{\boldsymbol{\alpha}}\right) \cdot \boldsymbol{\nu}-g_{R o b} u_{\boldsymbol{\alpha}} & =g_{N e u} & & \text { on } \Gamma_{N} \\
\left(-\boldsymbol{A}_{d i f} \nabla u_{\boldsymbol{\alpha}}-\boldsymbol{a}_{\text {con }} u_{\boldsymbol{\alpha}}\right) \cdot \boldsymbol{\nu} & =q_{i}+\alpha_{i} & & \text { on } \Gamma_{i}, i=1, \ldots, n .
\end{aligned}\right.
$$


Next, we aim at determining the appropriate value of the parameter $\boldsymbol{\alpha}$, for which the solution $u_{\boldsymbol{\alpha}}$ of (10) also satisfies the $n$ side conditions

$$
P_{i}\left(u_{\boldsymbol{\alpha}}\right) \equiv \int_{\Gamma_{i}} u_{\boldsymbol{\alpha}}=U_{i}, \quad i=1, \ldots, n .
$$

By the linearity of the integral operator $P_{i}$, we can write

$$
P_{i}\left(u_{\boldsymbol{\alpha}}\right)=P_{i}(v)+\sum_{j=1}^{n} \alpha_{j} P_{i}\left(z_{j}\right) .
$$

Hence, the conditions (11) give rise to the following linear algebraic system:

$$
\left(\begin{array}{ccc}
P_{1}\left(z_{1}\right) & \ldots & P_{1}\left(z_{n}\right) \\
\vdots & \ddots & \vdots \\
P_{n}\left(z_{1}\right) & \ldots & P_{n}\left(z_{n}\right)
\end{array}\right)\left(\begin{array}{c}
\alpha_{1} \\
\vdots \\
\alpha_{n}
\end{array}\right)=\left(\begin{array}{c}
U_{1}-P_{1}(v) \\
\vdots \\
U_{n}-P_{n}(v)
\end{array}\right)
$$

Now, we can prove the existence of a solution of the BVP (3).

Theorem 2 There exists a solution of the BVP (3).

Proof: We only need to show that the matrix $\boldsymbol{P}=\left(P_{i}\left(z_{j}\right)\right)_{i, j}$ of the linear algebraic system (12) is regular. To this end, assume that it is singular. Then at least one of its columns is a linear combination of the others. Without 
loss of generality we may suppose that it concerns the last column, i.e., that there exists a set of coefficients $\lambda_{1}, \ldots, \lambda_{n-1} \in \mathrm{R}$ such that

$$
P_{j}\left(z_{n}-\sum_{i=1}^{n-1} \lambda_{i} z_{i}\right)=0, \quad \text { for } j=1, \ldots, n \text {. }
$$

Introduce the function $w=z_{n}-\sum_{i=1}^{n-1} \lambda_{i} z_{i}$. On account of (8) and (13), this function is a solution of the following BVP:

$$
\left.\begin{array}{rlrl}
\nabla \cdot\left(-\boldsymbol{A}_{d i f} \nabla w-\boldsymbol{a}_{\text {con }} w\right)+a_{\text {sou }} w & =0 & & \text { in } \\
w & =0 & & \text { on } \Gamma_{D} \\
\left(-\boldsymbol{A}_{d i f} \nabla w-\boldsymbol{a}_{c o n} w\right) \cdot \boldsymbol{\nu}-g_{R o b} w & =0 & & \text { on } \Gamma_{N} \\
\left(-\boldsymbol{A}_{d i f} \nabla w-\boldsymbol{a}_{\text {con }} w\right) \cdot \boldsymbol{\nu} & =c_{i}(\mathrm{const}) & & \text { on } \Gamma_{i}, i=1, \ldots, n \\
P_{i}(w) & =0 & & i=1, \ldots, n .
\end{array}\right\}
$$

Clearly, this problem has the zero function as a trivial solution. However, by Theorem 1, this trivial solution is the only one. We conclude that

$$
z_{n}=\sum_{i=1}^{n-1} \lambda_{i} z_{i}
$$

We recall that the normal components of the flux of $z_{i}$ vanish on $\Gamma_{n}$, when $i \neq n$, see (8). Thus, (14) implies

$$
\left(-\boldsymbol{A}_{d i f} \nabla z_{n}-\boldsymbol{a}_{c o n} z_{n}\right) \cdot \boldsymbol{\nu}=0 \quad \text { on } \Gamma_{n},
$$


which contradicts the $\mathrm{BC}$ on $\Gamma_{n}$, imposed on $z_{n}$, see (8). Consequently, the matrix $\boldsymbol{P}$ is regular. The unique solution of (3) is then defined by the relation (9), where $\boldsymbol{\alpha}$ is the unique solution of the algebraic system (12).

We emphasize that the proof of Theorem 2 has a constructive character. First, one has to solve the well-posed auxiliary problems (7)-(8) with standard (i.e., pointwise) BCs. The desired solution will be an appropriate linear combination of the solutions of these auxiliary BVPs. The weights $\alpha_{1}, \ldots, \alpha_{n}$ of this linear combination are determined as the solution of a linear algebraic system. Moreover, the values $\alpha_{1}, \ldots, \alpha_{n}$ obtained determine the values of the normal component of the flux at the respective boundaries $\Gamma_{1}, \ldots, \Gamma_{n}$. Of course, also the initially unknown traces of the solution of (3) on $\Gamma_{1}, \ldots, \Gamma_{n}$, can now be evaluated from the constructed function $u_{\boldsymbol{\alpha}}$.

\section{Numerical experiments}

\subsection{First example}

Consider the geometrical situation shown in Figure 1a, corresponding, physically, to some soil venting facility, as mentioned in the introduction. The domain $\Omega$ is a circle with radius 2 , which has four circular holes with radius 0.2 . Here, $\Gamma_{P}$ represents a passive well (an opening to the atmosphere), while $\Gamma_{1}, \Gamma_{2}$ and $\Gamma_{3}$ represent active wells (pumps). Both the domain and the pas- 
sive well are centred at the origin $(0,0)$, while $\Gamma_{1}, \Gamma_{2}$ and $\Gamma_{3}$ are centred at $(1.5,0),(-1.2,0.5)$ and $(0,-1$.$) , respectively.$

We consider the following problem:

$$
\left\{\begin{aligned}
-\Delta u & =0 & & \text { in } \Omega \\
u & =0 & & \text { on } \Gamma_{P} \\
-\nabla u \cdot \boldsymbol{\nu} & =0 & & \text { on } \Gamma_{N} \\
-\nabla u \cdot \boldsymbol{\nu} & =c_{i} \text { (unknown constant) } & & \text { on } \Gamma_{i}, i=1,2,3 \\
\int_{\Gamma_{i}} u & =U_{i} & & i=1,2,3,
\end{aligned}\right.
$$

with $U_{1}=-0.1, U_{2}=-0.2$ and $U_{3}=-0.15$.

We apply the method of Section 3. As the BVP (7) is now completely homogeneous, we have $v=0$. For the approximation of the auxiliary problems (8), we set up a finite element method with continuous, piecewise linear polynomial functions on a triangular mesh on $\Omega$, consisting of 87552 triangles.

The resulting linear algebraic system of the type (12) has the solution

$$
\alpha_{1}=0.1565868721, \quad \alpha_{2}=0.3666216670, \quad \alpha_{3}=0.2920042558 .
$$

The contour lines for the corresponding solution $u_{\boldsymbol{\alpha}}$, cf. (9), are shown in Figure 1b.

To assess the accuracy of the method, we have - conversely-considered 

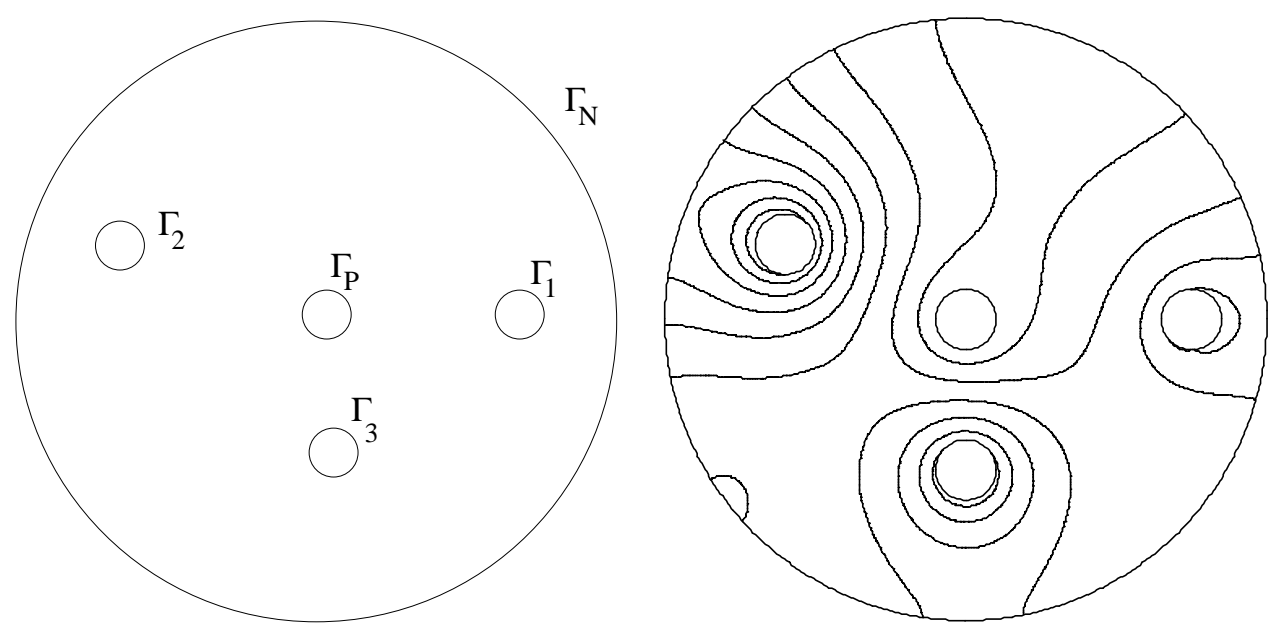

Figure 1: (a) The domain $\Omega$ and its boundary parts; (b) contour lines for $u_{\boldsymbol{\alpha}}$ 
the problem

$$
\left\{\begin{array}{rlrl}
-\Delta u & =0 & & \text { in } \\
u & =0 & & \text { on } \\
\Gamma_{P} \\
-\nabla u \cdot \nu & =0 & & \text { on } \Gamma_{N} \\
-\nabla u \cdot \nu & =\alpha_{i} & & \text { on } \Gamma_{i} \quad(i=1,2,3),
\end{array}\right.
$$

the values of $\alpha_{i}(i=1,2,3)$ being given by (16). This classical BVP is solved by the FEM mentioned above. When evaluating the line integrals over $\Gamma_{i}$ for the corresponding approximate solution, the values $U_{i},(i=1,2,3)$, imposed in (15), are recovered exactly.

In general, because of the proposed solution strategy, the error committed using FEMs for this type of problem will be of the same order as the one for a BVP with classical BCs.

\subsection{Second example}

Let $\Omega=(-1,1) \times(-1,1)$. The boundary $\partial \Omega$ is split into three parts $\Gamma_{D}, \Gamma_{N}$ and $\Gamma_{n}$, as shown in Figure 2. We consider the following BVP

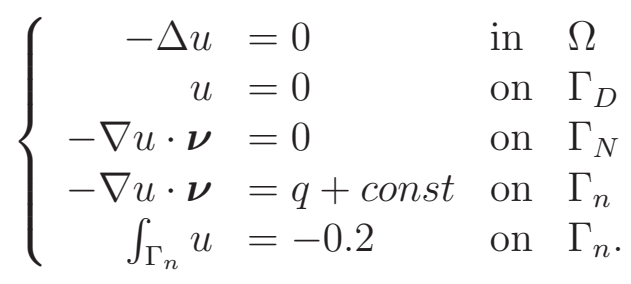




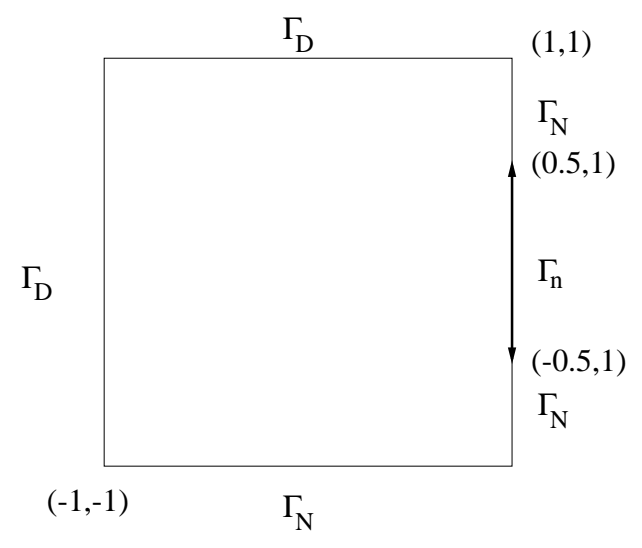

Figure 2: The domain $\Omega$ and its boundary parts 
The shape function $q$ either vanishes or takes the form $q(x, y)=1+y$. We compute the contour lines in both cases, in order to illustrate the influence of the shape function on the flow field in the vicinity of $\Gamma_{n}$. The geometry and the choice of the BCs may correspond to a vertical cross-section of an air pumping well in the soil. Such a rectangular domain is normally insulated from the bottom by an impervious layer, while it is open to the atmosphere at the top side. At a sufficiently large distance from the well (left side), one may assume a Dirichlet BC. The BCs on $\Gamma_{n}$ describe the inflow into the well, while the BCs on $\Gamma_{N}$ (right side) reflect some symmetry conditions.

We apply the method of Section 3. The auxiliary problems (7) and (8) are solved by a FEM with continuous, piecewise linear polynomials on an unstructured, triangular mesh with 1776 triangles. The contour lines for the solution are shown in Figure 3, for both cases considered. Although the average of the solutions on $\Gamma_{n}$ is the same, their behaviour is different, due to the shape function $q$, which prescribes the outflow on $\Gamma_{n}$.

Acknowledgements: This work was supported by the VEO-project no. 011 VO 697. The authors thank Roger Van Keer, coordinator of this project, for stimulating discussions and for his critical reading of the manuscript. 

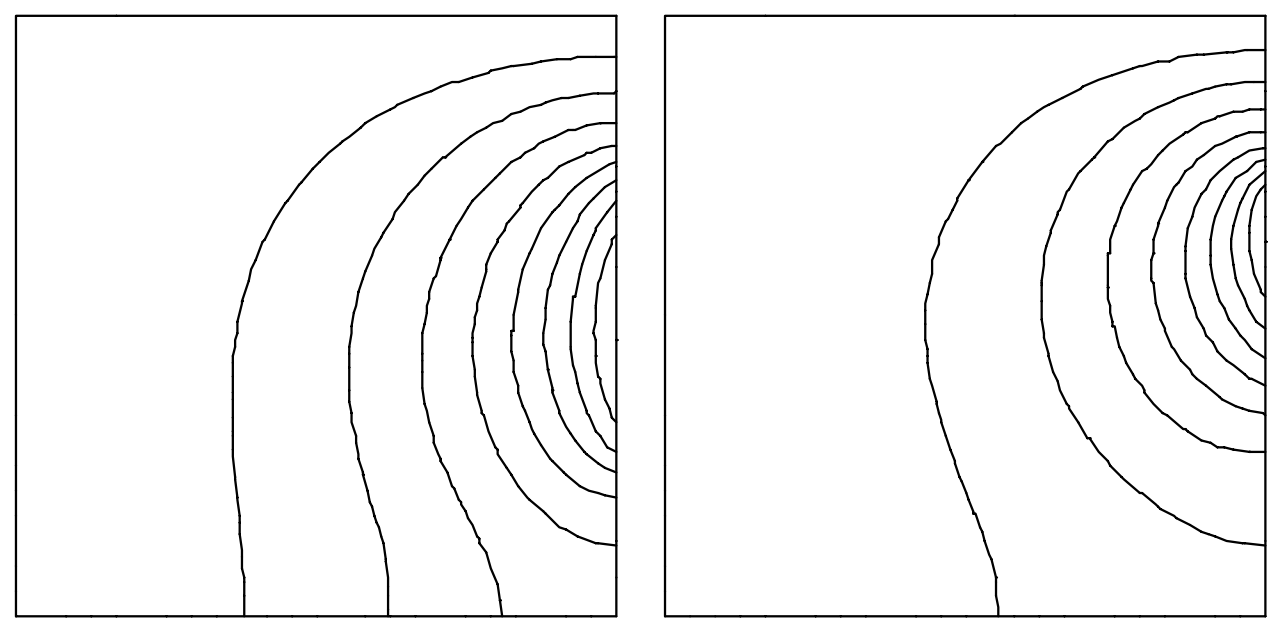

Figure 3: Contour lines for $u_{\boldsymbol{\alpha}}$ : (a) for $q(x, y)=0$; (b) for $q(x, y)=1+y$. 


\section{References}

[1] M. Authier et al. Problèmes variationnels linéaires. Régularité. In: R. Dautray and J.L. Lions, editors, Analyse mathématique et calcul numérique pour les sciences et les techniques, tome 1. Masson, Paris, 1985. C523

[2] H. Gerke et al. Optimal Control of Soil Venting: Mathematical Modeling and Applications, Vol.127, ISNM. Birkhäuser, 1999. C520, C520

[3] D. Gilbarg and N.S. Trudinger. Elliptic Partial Differential Equations of Second Order. Springer, Berlin, Heidelberg, 1983. C525

[4] R. Van Keer et al. Computational methods for the evaluation of the electromagnetic losses in electric machinery. Arch. Comp. Meth. Engrg., 5(4):385-443, 1999. C521 